the analysis was about the information of most recent year at baseline, which may not reflect the long-term sleeping habits.

Second, the lack of adjustments for other sleep factors in the analysis could have confounded their results. A plausible biological model, that is, light exposure at night (LAN)-melatonin-BC (Stevens and Davis, 1996) may interpret how poor sleep can directly affect the development of BC. In this hypothesis (Stevens and Davis, 1996), LAN is deemed to be associated with an increased risk for incident BC by decreasing the melatonin release by pineal gland. However, melatonin release rely on the light/dark cycle (Blask, 2009) rather than on sleep duration only, and other sleep characteristics such as sleep quality, LAN, the use of sleeping pills, habitual timing of sleep, and night waking times may also influence the outcome for incident BC (Yang et al, 2014); therefore, the potential confounding bias may exist. In addition, as the exposure data collection is described in a concise manner, it is unclear whether the 'sleep hours' is a real sleep duration at night-time or hours spent in bed.

Third, the findings, particularly for $\mathrm{ER}+\mathrm{PR}+\mathrm{BC}$, seemed to somewhat contradict the current possible biological mechanism, that is, LAN-melatonin-BC (Stevens and Davis, 1996). Such a discrepancy, as acknowledged by authors, could be due to chance. However, other factors, as mentioned above, including poor quality of exposure data (see comment 1) and lack of consideration for other sleep factors (see comment 2) may partly explain this controversy, because if the LAN-melatonin-BC hypothesis is true, as mentioned above, sleep is not necessarily required for synchronisation of the endogenous circadian rhythm (Blask, 2009), although melatonin release depends on a stable 24-h light/dark cycle, other sleep patterns such as habitual timing of sleep, waking up frequency, night-time lighting conditions, and sleep quality may also affect melatonin release (Yang et al, 2014).

Altogether, although this cohort study provided new information on the relationship between sleep duration and $\mathrm{BC}$ risk, the quality of exposure assessment and other covariates relating to sleep should be considered when interpreting results. With this in mind, we now are establishing a large population-based case-control study to assess the risk of $\mathrm{BC}$ associated with sleeping factors and other potential risk factors in Jiujiang City, China. As for exposure assessments, we are systematically collecting sleeping factors including sleep quality, LAN, night/shift work, the use of sleeping pills, sleep hours, habitual timing of sleep, and frequency for night-time wakings using the self-made 22-item sleeping factors questionnaire (SFQ). We have conducted a pilot study to check the validity and reproducibility of SFQ used in our project. In the pilot study, the SFQ was interview-administered twice, $\sim 1$ year apart, and participants were also asked to complete a 'sleeping diary' for 30 consecutive days every quarter over this same year accounting for seasonal effects. We examined the validation by comparing the average measures between two SFQs and four sleeping diaries, and examined the 1 -year stability of SFQ by comparing the measures in the two SFQs.

\section{ACKNOWLEDGEMENTS}

This study was supported by the National Natural Science Foundation of China (grant no. 81460514).

\section{CONFLICT OF INTEREST}

The authors declare no conflict of interest.

\section{DISCLAIMER}

The funders had no role in study design, data collection and analysis, decision to publish, or preparation of the manuscript.

\section{REFERENCES}

Blask DE (2009) Melatonin, sleep disturbance and cancer risk. Sleep Med Rev 13(4): 257-264.

Girschik J, Fritschi L, Heyworth J, Waters F (2012) Validation of self-reported sleep against actigraphy. J Epidemiol 22(5): 462-468.

Qian X, Brinton LA, Schairer C, Matthews CE (2014) Sleep duration and breast cancer risk in the breast cancer detection demonstration project follow-up cohort. Br J Cancer 112(3): 567-571.

Rothman KJ, Lash TL, Greenland S (2013) Modern Epidemiology. 3rd mid-cycle revised edn. (Lippincott Williams and Wilkins: Philadelphia, PA, USA.

Stevens RG, Davis S (1996) The melatonin hypothesis: electric power and breast cancer. Environ Health Perspect 104(Suppl 1): 135-140.

Yang WS, Deng Q, Fan WY, Wang WY, Wang X (2014) Light exposure at night, sleep duration, melatonin, and breast cancer: a dose-response analysis of observational studies. Eur J Cancer Prev 23(4): 269-276.

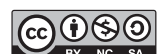

http://creativecommons.org/licenses/by-nc-sa/3.0/

\title{
Reply to 'Sleep duration and breast cancer risk in the breast cancer detection demonstration project follow-up cohort: true associations or bias?'
}

\author{
X Qian*,1, L A Brinton ${ }^{2}$, C Schairer ${ }^{3}$ and C E Matthews ${ }^{1}$
}

${ }^{1}$ Nutritional Epidemiology Branch, Division of Cancer Epidemiology and Genetics, National Cancer Institute, National Institutes of Health, Rockville, Maryland, USA; ${ }^{2}$ Hormonal and Reproductive Epidemiology Branch, Division of Cancer Epidemiology and Genetics, National Cancer Institute, National Institutes of Health, Rockville, Maryland, USA and ${ }^{3}$ Biostatistics Branch, Division of Cancer Epidemiology and Genetics, National Cancer Institute, National Institutes of Health, Rockville, Maryland, USA

Sir,

We thank Yang et al (2015) for their interest in and thoughtful review of our study. We agree with the correspondents that certain limitations should be considered when interpreting findings on sleep duration and breast cancer risk. As we acknowledged in our paper, self-reported sleep duration involving measurement error could lead to misclassification of our main exposure. We agree with Yang et al that misclassification can lead to bias in either direction. However, in our case, we believe the bias is more likely to be towards the null. A validation study by Lauderdale et al (2008) compared self-reported sleep duration to an objective measure (actigraphy), and found that the validity of self-reported sleep varied by the amount of sleep recorded. In general people tended to over-report their sleep duration, but the extent of over-reporting increased as sleep duration decreased. Therefore, short sleepers were at a higher risk of being misclassified as normal or long sleepers, which might have led to an inability to detect an increased risk of breast cancer among such individuals. Of course, regardless of the direction of the bias, misclassification of exposure is an important problem to consider, and we appreciate that Yang et al highlighted this particular element of our report. Moreover, we also agree with the correspondents that it is important to measure sleep at different time points in order to get a better estimate of long-term sleep duration, and to consider other sleep characteristics and sleep-related factors, such as sleep quality and exposure to light at night.

We are aware of the discrepancy between our findings and the melatonin hypothesis, which suggests that short sleep duration is associated with decreased levels of melatonin. Because melatonin is a molecule with anti-oestrogenic effects, decreased levels of melatonin may increase the risk of ER + tumours (Blask, 2009). We found no association between sleep duration and hormone receptor-positive tumours, which is consistent with the only two studies that examined sleep duration in relation to breast cancer subtypes defined by hormone receptor status. Notably, both studies, an Australian case-control study and a study in the Women's Health Initiative, showed no relationship between sleep duration and ER + tumours (Girschik et al, 2013; Vogtmann et al, 2013). In contrast, we found an increased risk associated with short sleep durations for hormone receptor-negative breast cancers. Although we cannot exclude the possibility that our finding is due to bias or chance, we believe that there are biological mechanisms that support this observed association. For example, short sleep and sleep deprivation have been associated with factors that may influence breast cancer risk 
independent of oestrogen pathways, such as metabolic dysfunction (Gangwisch et al, 2007) and chronic inflammation (Irwin et al, 2006).

Again, we thank Yang et al for this letter and are glad that more studies, such as the population-based case-control study in Jiujiang city mentioned by Yang et al, are using objective measures along with questionnaires to better assess both the quantity and quality of sleep in relation to breast cancer risk and other health outcomes.

\section{REFERENCES}

Blask DE (2009) Melatonin, sleep disturbance and cancer risk. Sleep Med Rev 13(4): 257-264.

Gangwisch JE, Heymsfield SB, Boden-Albala B, Buijs RM, Kreier F, Pickering TG et al. (2007) Sleep duration as a risk factor for diabetes incidence in a large U.S. sample. Sleep 30(12): 1667-1673.
Girschik J, Heyworth J, Fritschi L (2013) Self-reported sleep duration, sleep quality, and breast cancer risk in a population-based case-control study. Am J Epidemiol 177(4): 316-327.

Irwin MR, Wang M, Campomayor CO, Collado-Hidalgo A, Cole S (2006) Sleep deprivation and activation of morning levels of cellular and genomic markers of inflammation. Arch Intern Med 166(16): 1756-1762.

Lauderdale DS, Knutson KL, Yan LL, Liu K, Rathouz PJ (2008) Self-reported and measured sleep duration: how similar are they? Epidemiology 19(6): $838-845$.

Vogtmann E, Levitan EB, Hale L, Shikany JM, Shah NA, Endeshaw Y et al. (2013) Association between sleep and breast cancer incidence among postmenopausal women in the women's health initiative. Sleep 36(10): 1437-1444.

Yang W-S, Wang X, Deng Q, Zhao H, Fan W-Y (2015) Sleep duration and breast cancer risk in the breast cancer detection demonstration project follow-up cohort: true associations or bias? Br J Cancer 112(11): 1838-1839.

${ }^{\star}$ Correspondence: Dr Q Xiao; E-mail: qian.xiao@nih.gov

Published online 21 April 2015

(c) 2015 Cancer Research UK. All rights reserved 0007-0920/15

http://creativecommons.org/licenses/by-nc-sa/3.0/

\section{Comment on 'Possible pro-carcinogenic association of endotoxin on lung cancer among Shanghai women textile workers'}

R Rylander ${ }^{\star}, 1$ and R Jacobs ${ }^{2}$

${ }^{1}$ BioFact Environmental Health Research Center, Lerum, Sweden and ${ }^{2}$ Environmental and Occupational Health Sciences, School of Public Health and Information Sciences, University of Louisville, Louisville, KY, USA

Sir,

In a recent article in this Journal, Checkoway et al (2014) suggest that the exposure to endotoxin in industrial environments is associated with an increase in the risk of lung cancer.

A number of studies over the past 50 years has demonstrated a decreased risk in different environments involving a high exposure to endotoxin such as cotton handling and farming (Rylander, 1992; Maestrangelo et al, 2005; Lenters et al, 2010). Plausible cellular mechanisms for this defence have been discussed. In the data now presented there are no significant differences in risk-all are within the 95\% confidence limit-and no significance for trend in relation to exposure duration. The only observation, thoroughly discussed, is a small, non-significant increase in risk in a subgroup. It is difficult to understand how such data can be used as a support to challenge a previously well-established relationship.

More serious is the lack of control of possible confounding factors. It is well known that indoor air pollution from cooking fuels is a risk factor for lung cancer. Such exposures change over the years and are closely related to socio-economic factors. The problem is discussed but in the absence of data the discussion remains speculative. Diet modulates the risk of lung cancer but is not discussed (Seow et al, 2002; Rylander and Axelsson, 2006). Finally, possible changes in endotoxin exposure over the years are not dealt with. Also in China, work hygiene standards have improved over the years since the measurements were made and could result in a change of exposure to endotoxin.

In view of the above, a correct conclusion from the material presented is that 'no relation between endotoxin exposure and lung cancer risk could be detected'.

\section{REFERENCES}

Checkoway H, Lundin JI, Costello S, Ray R, Li W, Eisen EA, Astrakianakis G, Seixas N, Applebaum K, Gao DL, Thomas DB (2014) Possible pro-carcinogenic association of endotoxin on lung cancer among Shanghai women textile workers. Br J Cancer 111: 603-607.

Lenters V, Basinas I, Beane-Freeman I, Boffetta P, Checkoway H, Coggon D, Portengen L, Sim M, Wouters IM, Heederik D, Vermeulen R (2010) Endotoxin exposure and lung cancer risk: a systematic review and meta-analysis of the published literature on agriculture and cotton textile workers. Cancer Causes Control 21: 523-555.

Maestrangelo G, Grange J, Fadda E, Fedeli U, Buja A, Lange JH (2005) Lung cancer risk effect of dairy farming and the consequence of removing occupational exposure. Am J Epidemiol 161: 1037-1046.

Rylander R (1992) Environmental exposures with decreased risk for lung cancer? Int J Epidemiol 19: S67-S71.

Rylander R, Axelsson G (2006) Lung cancer risk in relation to vegetable and fruit consumption and smoking. Int J Cancer 118: 739-743.

Seow A, Poh WT, The M, Eng P, Wang YT, Tan WC, Chia KS, Yu M, Lee HP (2002) Diet, reproductive factors, and lung cancer risk among Chinese women in Singapore: evidence for a protective effect of soy in non-smokers. Int J Cancer 97: 365-371.

\section{Reply to Comment on: 'Possible pro-carcinogenic association of endotoxin on lung cancer among Shanghai women textile workers'}

H Checkoway ${ }^{\star}, 1$, J I Lundin², S Costello ${ }^{3}$, R M Ray ${ }^{4}$, W Li ${ }^{4}$, E A Eisen ${ }^{5}$, G Astrakianakis ${ }^{6}$, K Applebaum ${ }^{7}$, D L Gao ${ }^{8}$ and D B Thomas ${ }^{4}$

${ }^{1}$ Department of Family and Preventive Medicine, University of California, San Diego, La Jolla, CA 92093, USA; ${ }^{2}$ Department of Environmental and Occupational Health Sciences, University of Washington, Seattle, WA, USA; ${ }^{3}$ Department of Environmental Health Sciences, University of California, Berkeley, Berkeley, CA, USA; ${ }^{4}$ Division of Public Health Sciences, Fred Hutchinson Cancer Research Center, Seattle, WA 98109, USA; ${ }^{5}$ epartment of Environmental Health Sciences, University of California, Berkeley, Berkeley, CA, USA; ${ }^{6}$ School of Population and Public Health, University of British Columbia, Vancouver, BC V6T1Z4, Canada; ${ }^{7}$ Department of Environmental and Occupational Health, George Washington University, Washington, DC 20052, USA and ${ }^{8}$ Zhong Shan Hospital Cancer Center, Shanghai 2000030, China

Sir,

We appreciate the thoughtful comments by Rylander and Jacobs (2015) on our paper (Checkoway et al, 2014). The absence of an inverse exposure-response relation for endotoxin and lung cancer in the extended follow-up was somewhat unexpected in view of the reported consistent findings from numerous prior studies, including our initial follow-up of the 